\title{
Vital signs effectively predict non-hypoglycaemia in patients with altered mental status in pre-hospital settings: A retrospective analysis
}

\section{Daisuke Mizu ( $\nabla$ dct_water@yahoo.co.jp )}

Kobe City Medical Center General Hospital: Kobe Shiritsu Iryo Center Chuo Shimin Byoin https://orcid.org/0000-0002-0586-6159

\section{Yoshinori Matsuoka}

Kobe City Medical Center General Hospital: Kobe Shiritsu Iryo Center Chuo Shimin Byoin

Ji Young Huh

Kobe City Medical Center General Hospital: Kobe Shiritsu Iryo Center Chuo Shimin Byoin

\section{Koichi Ariyoshi}

Kobe City Medical Center General Hospital: Kobe Shiritsu Iryo Center Chuo Shimin Byoin

\section{Original research}

Keywords: pre-hospital emergency care, vital signs, hypoglycaemia, impaired consciousness

Posted Date: October 12th, 2020

DOl: https://doi.org/10.21203/rs.3.rs-87519/v1

License: (9) (1) This work is licensed under a Creative Commons Attribution 4.0 International License. Read Full License 


\section{Abstract}

\section{Background}

Blood glucose (BG) measurement by paramedics for patients with altered mental status is recommended as pre-hospital care in Japan. Therefore, paramedics prioritise BG measurement over transport to hospital even in emergency conditions such as hypotension or hypoxaemia. The purpose of this study was to examine the relationship between BG levels and vital signs, and to evaluate whether vital signs are effective in determining the necessity of BG measurement in pre-hospital settings.

\section{Methods}

We extracted data of patients who had BG measurements performed by paramedics in Kobe City from April 2015 to March 2019. We retrospectively investigated patient age, sex, presence of hypoglycaemia (BG level $<50 \mathrm{mg} / \mathrm{dL}$ ) and vital signs. If a patient did not have hypoglycaemia and was transported to the Kobe City Medical Centre General Hospital, a final diagnosis was obtained. Patients aged below 15 years, and those with BG measurement errors, missing vital sign data, or a Japan Coma Scale 0 and I-digit codes were excluded. The $\chi 2$ test and Mann-Whitney $U$ test were used for statistical analysis, and $P<0.05$ was considered statistically significant.

\section{Results}

Of the 1,791 patients, 1,242 were eligible for analysis (mean age, 71.9 years; 805 [58\%] male). Hypoglycaemia was observed in 324 patients (26.1\%). Of the 918 non-hypoglycaemic patients, 253 $(27.6 \%)$ were transported to our hospital and stroke was the most common final diagnosis (61 patients [24.1\%]). The non-hypoglycaemic group had more elderly patients than the hypoglycaemic group (median 73 vs. 76 years; $P<0.01$ ). A significant difference in each vital sign were noted between hypoglycaemic and non-hypoglycaemic groups, with body temperature showing the highest difference between groups (area under the curve, $0.71 ; 95 \%$ confidence interval [Cl], 0.68-0.74). Furthermore, in cases with systolic blood pressure being over $100 \mathrm{mmHg}$ and body temperature being $38^{\circ} \mathrm{C}$ or less, it was highly unlikely that hypoglycaemia caused impaired consciousness (likelihood ratio 0.12 and $0.16 ; 95 \% \mathrm{Cl} 0.05-0.25$ and 0.06-0.35, respectively).

\section{Conclusion}

When considering pre-hospital hypoglycaemia assessment, vital signs are an effective index. If patients have significant hypotension or high fever, paramedics should consider immediate transport rather than BG measurement.

\section{Background}

Hypoglycaemia is a condition that must be considered in patients with altered mental status (AMS); it can be easily diagnosed by measuring blood glucose (BG) levels. In January 2014, Japanese paramedics 
were officially permitted to measure BG levels in patients with impaired consciousness. In confirmed cases of hypoglycaemia, paramedics are allowed to administer glucose through an intravenous line. Conversely, hypoglycaemia is not a common condition in pre-hospital settings. Only $1-5 \%$ of emergency transport patients have severe hypoglycaemia that requires immediate treatment [1,2], and only $5 \%$ of comatose patients had a metabolic cause such as hypoglycaemia [3]. Thus, paramedics often perform unnecessary BG measurements in patients with AMS because there are no clear criteria for measuring BG other than the consciousness level. In patients with AMS, paramedics need to consider more critical medical conditions such as stroke, seizures, and sepsis at the top of differential diagnoses. In such medical emergencies, BG measurement may be damaging because it delays transport and the time to appropriate treatment in the hospital.

Although past medical history of diabetes mellitus and neurological examination findings provide useful clinical information to determine whether hypoglycaemia is a potential cause of AMS $[1,4,5]$, it is often difficult to obtain a detailed medical history and perform physical examination on the field, especially for patients with AMS. Therefore, findings of any simple, routine physical examination, such as vital signs, can be used to safely exclude patients with AMS who do not need BG measurement. This would be a very useful pre-hospital emergency parameter and may effectively save time for patients who may need early transport more than BG measurement. Although there have been few studies regarding the changes in vital signs in hypoglycemic patients [6-8], there have been no studies regarding a correlation between vital signs and the need for BG measurement in pre-hospital settings. In this study, we examined whether vital signs are effective indicators for determining the need for BG measurement in patients with AMS in pre-hospital settings.

\section{Methods}

\section{Study design and population}

We performed a retrospective study based on data extracted from the Kobe City Fire Department (KCFD) database. Our study population included patients with AMS for whom paramedics performed BG measurements from April 2015 to March 2019. This study was approved by the ethics board of the Kobe City Medical Centre General Hospital.

Paramedics performed BG measurements according to the protocol shown in Table 1. We collected the following data: age, sex, Japan Coma Scale (JCS), presence of hypoglycaemia (defined by KCFD as < $50 \mathrm{mg} / \mathrm{dL}$ ), vital signs (systolic blood pressure [sBP], heart rate [HR], respiratory rate [RR], oxygen saturation by pulse oximetry $\left[\mathrm{SpO}_{2}\right]$ and body temperature $\left.[\mathrm{BT}]\right)$. We excluded patients under 15 years of age with BG measurement errors or missing data for any vital sign and JCS. We also excluded patients who had BG measurements taken despite their conscious levels outside the BG measurement protocol (for example, JCS 0, I-digit code). In Japan, JCS is used as a scale for evaluating patient consciousness (level 9) and is also used in BG measurement protocols for paramedics. JCS comprises four categories: 0

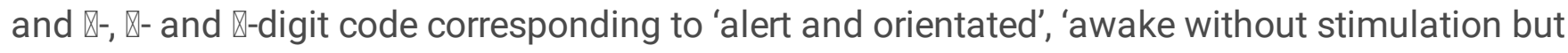


disoriented', 'awake with stimulation' (return to previous state after cessation of stimulation) and 'unarousable by any painful stimulation', respectively. Each code has three subcategories: 1, 2 and 3 in

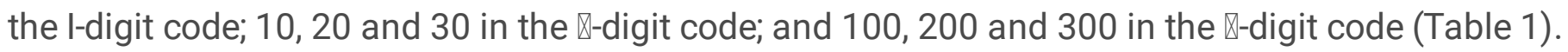

Table 1. Kobe City Fire Department blood glucose measurement criteria and Japan Coma

\section{Blood glucose measurement protocol}

Patients who satisfy both of the following two conditions:

1. Recognised impaired consciousness (as a guide $\geqq$ Japan Coma Scale $₫-10$ )

2. There is an advantage in identifying consciousness disorders and selecting a hospital by performing blood glucose measurement

However, if subarachnoid haemorrhage is strongly suspected (and pain stimulation due to skin puncture for blood glucose measurement is considered inappropriate for the patient), it is excluded.

\begin{tabular}{|l|l|}
\hline Level & Grade \\
\hline Alert & 0 \\
\hline The patient is awake without any stimuli, and: & I-digit code \\
\hline - almost fully conscious & 1 \\
\hline - unable to recognise time, place, and person & 2 \\
\hline - unable to recall name or date of birth & 3 \\
\hline The patient can be aroused and then revert to previous state after ending stimuli & II-digit code \\
\hline - easily with a normal call & 10 \\
\hline - with a loud voice or by shaking shoulders & 20 \\
\hline - only with repeated painful stimuli and calls & 30 \\
\hline The patient cannot be aroused with any painful stimuli, and: & III-digit code \\
\hline - responds with movements to avoid the stimuli & 100 \\
\hline - responds with slight movements including a decerebrate and decorticate posture & 200 \\
\hline - fails to respond & 300 \\
\hline
\end{tabular}

\section{Outcome}

The primary outcome of this study was that it showed the effectiveness of each vital sign as a hypoglycaemia predictor in AMS patients.

\section{Statistical analysis}


We compared the differences in vital signs between the hypoglycaemic and non-hypoglycaemic groups. The $\chi 2$ and Mann-Whitney $U$ tests were used, and a P-value higher than 0.05 was considered significant. The effectiveness of each vital sign in predicting hypoglycaemia was analysed using receiver operating characteristic (ROC) curves, and the area under a curve (AUC) was calculated to estimate diagnostic accuracy. The sensitivity, specificity and stratum-specific likelihood ratio (SSLR) were then calculated to examine the cut-off value of each vital sign. Statistical analysis was performed using JMP version 12 (SAS Institute Inc).

\section{Results}

During the study period, the paramedics performed BG measurements on 1791 patients; of these, 431 patients were excluded (three patients under 15 years of age, two patients with errors in BG level measurements, and 426 patients with missing vital signs or JCS data). From these 1,360 patients, we excluded 118 patients because they had BG measurements outside the protocol (15 patients with a JCS

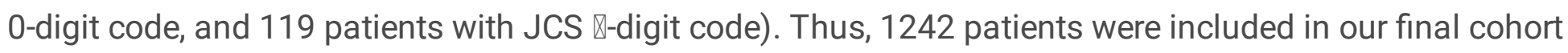
(Figure 1).

In total, 324 patients (26.1\%) had hypoglycaemia. Table 2 shows patient characteristics according to the presence or absence of hypoglycaemia. Compared with non-hypoglycaemic patients, hypoglycaemic patients were significantly younger (median age 73 years [interquartile range (IQR), 62-82] vs. 76 years [IQR, 65-84]; P< 0.01).

Table 2. Characteristics of the patients 


\begin{tabular}{|c|c|c|c|}
\hline & Hypoglycaemia & Non-hypoglycaemia & P-value \\
\hline $\mathrm{N}$ & 324 & 918 & \\
\hline Male n, (\%) & $191(59.0)$ & $529(57.6)$ & 0.69 \\
\hline Age, median $\left(\mathrm{IQR}^{1}\right)$ & $73(62-82)$ & $76(65-84)$ & $*<0.01$ \\
\hline $\mathrm{sBP}^{2}(\mathrm{mmHg})$, median (IQR) & $150(129-170)$ & $136(110-164)$ & $*<0.01$ \\
\hline $\mathrm{HR}^{3}$ (/min), median (IQR) & $80(70-91.3)$ & $82(70-100)$ & *0.03 \\
\hline $\mathrm{RR}^{4}(/ \mathrm{min})$, median (IQR) & $20(18-20)$ & $20(18-24)$ & $\star 0.03$ \\
\hline $\mathrm{SpO}_{2}^{5}(\%)$, median (IQR) & $96(95-98)$ & $96(93-98)$ & $*<0.01$ \\
\hline $\mathrm{BT}^{6}\left({ }^{\circ} \mathrm{C}\right)$, median (IQR) & $36(35.5-36.4)$ & $36.5(36.0-37.0)$ & $*<0.01$ \\
\hline \multicolumn{4}{|l|}{$\mathrm{JCS}^{7} \mathrm{n},(\%)$} \\
\hline II-digit code & $198(61.1)$ & $597(65.0)$ & 0.21 \\
\hline III-digit code & $126(38.9)$ & $321(35.0)$ & 0.21 \\
\hline \multicolumn{4}{|c|}{$\begin{array}{l}{ }^{1} \text { interquartile range; }{ }^{2} \text { systolic blood pressure; }{ }^{3} \text { heart rate; }{ }^{4} \text { respiratory rate; }{ }^{5} \text { oxygen saturation by } \\
\text { pulse oximetry; }{ }^{6} \text { body temperature; }{ }^{7} \text { Japan coma scale; }\end{array}$} \\
\hline
\end{tabular}

\section{Examination of vital signs of hypoglycaemia and non-hypoglycaemia patients}

There were significant differences in all vital signs between the two groups, and the distribution of each vital sign is shown in Figure 2. The ROC curves of these vital signs to predict hypoglycaemia in AMS patients are shown in Figure 3. The AUCs for each vital sign were calculated, and were $0.61(95 \% \mathrm{Cl}$ : 0.58-0.64) for sBP, 0.54 (95\% Cl: $0.51-0.57)$ for HR, 0.54 (95\% Cl: $0.51-0.58)$ for RR, 0.57 ( $95 \%$ Cl: $0.54-$ $0.61)$ for $\mathrm{SpO}_{2}$, and 0.71 (95\% Cl: $\left.0.68-0.74\right)$ for BT. The sensitivity, specificity and SSLR of each stratified vital sign are shown in Table 3. When sBP was more than $100 \mathrm{mmHg}$ or BT was $38^{\circ} \mathrm{C}$ or less, the likelihood ratio (LR) was 0.12 (95\% Cl: $0.05-0.25)$ and 0.15 (95\% Cl: $0.06-0.35)$, respectively.

\section{Details of non-hypoglycaemic patients}

Overall, 253 non-hypoglycaemic patients had a final diagnosis recorded (Table 4). Stroke was the most common diagnosis $(24.1 \%$ ) and other emergency medical conditions including poisoning (12.3\%), infectious diseases (10.7\%) and seizure (9.9\%) comprised more than half the cases. Nine patients (3.6\%) were diagnosed with hypoglycaemia as the final cause, even though their BG level at their pre-hospital assessment was greater than $50 \mathrm{mg} / \mathrm{dL}$. 
Table 3. Stratum-specific sensitivity, specificity, and likelihood ration of each vital sign 


\section{Number of patients}

Strata Hypoglycaemia Non-hypoglycaemia Sensitivity Specificity $\operatorname{LR}^{1}(95 \% \mathrm{Cl})$ $\mathrm{sBP}^{2}(\mathrm{mmHg})$

$<100$

100-119 36

146

0.98

0.16

$0.15(0.05-0.25)$

120-139

79

161

0.87

0.34

$0.63(0.45-0.88)$

140-159 81

160-179 65

$\geqq 180$

57

176

0.63

0.53

1.27 (1.00-1.60)

$\mathrm{HR}^{3}$ (/min)

$<60 \quad 18$

$60-70$

80-99

18

166

0.34

0.34

1.38 (1.06-1.74)

100-119

125

142

0.18

0.18

1.30 (0.99-1.68)

127

0.00

1.00

1.27 (0.95-1.68)

$120-139$
$\mathrm{RR}^{4}(/ \mathrm{min})$

\begin{tabular}{|llllll|}
\hline$<10$ & 0 & 3 & 1.00 & 0.00 & $0.00(0.00-3.61)$ \\
\hline $10-19$ & 134 & 350 & 0.59 & 0.39 & $1.08(0.92-1.26)$ \\
\hline $20-29$ & 164 & 446 & 0.08 & 0.87 & $1.04(0.92-1.18)$ \\
$\geqq 30$ & 26 & 119 & 0.00 & 1.00 & $0.62(0.41-0.92)$ \\
\hline
\end{tabular}

$\mathrm{SpO}_{2}{ }^{5}(\%)$

\begin{tabular}{|llllll}
$<90$ & 22 & 118 & 0.93 & 0.13 & $0.53(0.34-0.81)$ \\
$90-93$ & 39 & 130 & 0.81 & 0.27 & $0.85(0.61-1.18)$ \\
$94-97$ & 152 & 436 & 0.34 & 0.75 & $0.99(0.86-1.12)$ \\
$\geqq 98$ & 111 & 234 & 0.00 & 1.00 & $1.34(1.11-1.61)$ \\
$\mathrm{BT}^{6}\left({ }^{\circ} \mathrm{C}\right)$ & & & & & \\
$<35.0$ & 36 & 36 & 0.89 & 0.04 & $2.83(1.82-4.40)$
\end{tabular}




\begin{tabular}{|llllll|}
\hline $35.0-35.9$ & 112 & 146 & 0.54 & 0.20 & $2.17(1.76-2.67)$ \\
\hline $36.0-36.9$ & 153 & 489 & 0.07 & 0.73 & $0.89(0.78-1.01)$ \\
\hline $37.0-37.9$ & 18 & 151 & 0.02 & 0.90 & $0.34(0.21-0.54)$ \\
$\geqq 38.0$ & 5 & 96 & 0.00 & 1.00 & $0.15(0.06-0.35)$ \\
\hline $\begin{array}{l}{ }^{1} \text { likelihood ratio; }{ }^{2} \text { systolic blood pressure; }{ }^{3} \text { heart rate; }{ }^{4} \text { respiratory rate; }{ }^{5} \text { oxygen saturation by pulse } \\
\text { oximetry; }{ }^{6} \text { body temperture }\end{array}$ & & & \\
\hline
\end{tabular}

Table 4. Final diagnosis of non-hypoglycaemic patients transferred to the Kobe City Medical Centre General Hospital

\begin{tabular}{|ll|}
\hline Final diagnosis & $\mathrm{n},(\%)$ \\
\hline Hypoglycaemia & $9(3.6)$ \\
\hline Stroke & $61(24.1)$ \\
\hline Toxin & $31(12.3)$ \\
\hline Seizure & $27(10.7)$ \\
\hline Syncope & $25(9.9)$ \\
\hline Metabolic \& electrolyte (other than hypoglycaemia) & $21(8.3)$ \\
\hline Psychiatry & $15(5.9)$ \\
\hline Traumatic brain injury & $12(4.7)$ \\
\hline Unknown & $11(4.3)$ \\
\hline Other causes & $17(6.7)$ \\
\hline Hypothermia or heat illness & $24(9.5)$ \\
\hline Acute heart failure & $10(41.7)$ \\
\hline Acute aortic dissection & $4(16.7)$ \\
\hline Dehydration & $3(12.5)$ \\
\hline Gastrointestinal bleeding & $3(12.5)$ \\
\hline CO ${ }_{2}$ narcosis & $2(8.3)$ \\
\hline Hypertensive encephalopathy & $1(4.2)$ \\
\hline
\end{tabular}




\section{Discussion}

This retrospective study examined the effectiveness of vital signs to predict hypoglycaemia in patients presenting with AMS to avoid unnecessary BG measurements by paramedics attending emergency medical conditions in pre-hospital settings. In this study, there were statistically significant differences in all vital signs between non-hypoglycaemic and hypoglycaemic patients. Among the vital signs evaluated, sBP and BT may be effective in predicting hypoglycaemia, and AUC indicated that BT could be the most useful vital sign for differentiating between hypoglycaemic and non-hypoglycaemic episodes.

In our study, the hypoglycaemic group had more abnormal vital signs than the non-hypoglycaemic group. Wide variability in bioprotective responses can occur in hypoglycaemia. Such variabilities include but not limited to decreased insulin secretion, increased glucagon, epinephrine, and norepinephrine secretion associated with the activation of the sympathetic-adrenal function system. These responses occur early in the course of low BG levels and cause an increase in SBP, RR and HR [6-8]. Our study findings were consistent with these previous studies, and the hypoglycaemic group tended to have increased SBP, RR and HR compared to the non-hypoglycaemic group. In terms of the association between hypoglycaemia and $\mathrm{BT}$, we found that the higher the $\mathrm{BT}$, the lower the likelihood of hypoglycaemia. There have been reports that hypoglycaemia is associated with hypothermia [9], which appeared to be induced by glucose deficiency in the cells of the hypothalamic centre regulating BT [10].

Among these vital sign changes, $\mathrm{SBP}$ and $\mathrm{BT}$ may be the most useful indicators for predicting hypoglycaemia in our study. When sBP was less than $100 \mathrm{mmHg}$ and BT was more than $38^{\circ} \mathrm{C}$, the LRs of hypoglycaemia were very low ( 0.12 and 0.15 , respectively). This finding is supported by previous studies. Ikeda et al. reported that high sBP was associated with brain lesions accounting for impaired consciousness observed in patients with AMS [11], and higher BT was associated with infectious diseases such as sepsis in other studies $[12,13]$.

Although early detection and treatment of hypoglycaemia are important, previous studies have found that BG measurement prolongs the field time of emergency services by $2-5$ minutes in Japan $[14,15]$. This delay can be fatal and may be damaging in patients with impaired consciousness with other critical conditions such as stroke or sepsis as found in our study. Therefore, it is ideal to avoid unnecessary BG measurement in these patients and transfer them to the hospital as early as possible.

Evaluating the known risk factors for hypoglycaemia, such as history of diabetes mellitus and renal dysfunction, is useful to predict it [16-18], though impaired consciousness makes it difficult to obtain clinical information from patients. Vital signs represent important information that can be obtained over a short period of time even in patients with impaired consciousness, and the results of our study indicated that certain changes in vital signs such as $\mathrm{sBP}<100 \mathrm{mmHg}$ or $\mathrm{BT} \geq 38^{\circ} \mathrm{C}$ may help paramedics to make decisions to not to perform BG measurement and avoid prolonged pre-hospital care. In this study, nine patients in the non-hypoglycaemic group were diagnosed hypoglycaemia. The BG levels of eight patients in pre-hospital settings were $50-65 \mathrm{mg} / \mathrm{dL}$, This indicated an undeniable possibility of hypoglycaemia. One patient was found at the history interview to have been administered 
oral glucose by a family member before the paramedic performed BG measurement. Based on these results, it may be necessary to discuss the validity of the hypoglycaemia criterion hypoglycaemia as less than $50 \mathrm{mg} / \mathrm{dl}$. In contrast, these nine patients did not show vital signs ( $\mathrm{SBP}<100 \mathrm{mmHg}, \mathrm{BT} \geq 38^{\circ} \mathrm{C}$ ) that would indicate that the cause of AMS is likely to be non-hypoglycaemic, and we believe that the validity of the results of this study will not change significantly.

\section{Limitations}

This study has several limitations. First, the number of patients with AMS who did not receive BG measurements, despite satisfying the criteria for the BG measurement protocol, was highly dependent on the paramedics' choice on whether to perform a BG measurement. It could not have been determined which patients were transported to the hospital without BG measurement. Because brain injuries such as stroke are considered an emergency and are less likely to be associated with hypoglycaemia [5], BG measurement may not have been performed if the paramedics suspected such diseases. Patients with brain injury often develop fever and hypertension [11, 19], and the results of our study would not have been significantly different from the present results even if they had been monitored. Second, in this study, the possibility of hypoglycaemia may not have been adequately assessed by the paramedics with regard to patient background including past medical history and medications, which may have influenced the decision to perform BG measurement. Further, there was a bias in patient selection, though we think that this is a practical consequence of paramedical field activities. Finally, the final diagnosis other than hypoglycaemia was recorded only for those patients transported to a single institution, and the possibility of comorbidities other than hypoglycaemia was not sufficiently examined. Thus, further investigations, including enrolling cases from other institutions, is necessary.

\section{Conclusion}

In distinguishing hypoglycaemia from non-hypoglycaemia in pre-hospital settings, high fever and low sBP were more effective than other vital signs. In particular, when BT was $\geq 38^{\circ} \mathrm{C}$ or SBP was $<$ $100 \mathrm{mmHg}$, the possibility of hypoglycaemia was very low, and paramedics should consider early transport rather than BG measurements.

\section{Abbreviations}

BG

blood glucose

AMS

Altered Mental Status

JCS

Japan Coma Scale

BT

body temperature 
sBP

systolic blood pressure

$\mathrm{HR}$

heart rate

RR

respiratory rate

$\mathrm{SpO}_{2}$

oxygen saturation by pulse oximetry

KCFD

Kobe City Fire Department

ROC

receiver operating characteristic

AUC

area under the curve

$\mathrm{Cl}$

confidence interval

LR

likelihood ratio

SSLR

stratum-specific likelihood ratio

\section{Declarations}

\section{Ethics Approval}

This study was performed according to Helsinki Declarations and approved by the ethics board of the Kobe City Medical Centre General Hospital (registration number: zn190919). The need for informed consent was waived by the review board due to the retrospective nature of this study.

\section{Consent for publication}

Not applicable

\section{Availability of data and materials}

The datasets used and/or analysed during the current study are available from the corresponding author on reasonable request.

\section{Competing interests}

The authors declare that they have no competing interests.

\section{Funding}


The authors received no financial support for the research, authorship, and/or publication of this article.

\section{Authors contribution}

MD, MY, and HJ designed the study. MD acquired the data. MD and MY analysed and interpreted the data. MD, MY, HJ, and AK prepared and revised the manuscript.

\section{Acknowledgments}

We would like to thank Tatsuya Nakata and other members of Kobe City Fire Department for collecting data. We also thank Editage (www.editage.com) for English language editing.

\section{References}

1. Tsujimoto T, Yamamoto-Honda R, Kajio H, Kishimoto M, Noto H, Hachiya R, et al. Prediction of 90-day mortality in patients without diabetes by severe hypoglycemia: blood glucose level as a novel marker of severity of underlying disease. Acta Diabetol. 2015;52:307-14.

2. Parsaik AK, Carter RE, Pattan V, Myers LA, Kumar H, Smith SA, et al. Population-based study of severe hypoglycemia requiring emergency medical service assistance reveals unique findings. J Diabetes Sci Technol. 2012;6:65-73.

3. Forsberg S, Höjer J, Ludwigs U. Prognosis in patients presenting with non-traumatic coma. J Emerg Med. 2012;42:249-53.

4. Sinclair JE, Austin M, Froats M, Leduc S, Maloney J, Dionne R, et al. Characteristics, prehospital management, and outcomes in patients assessed for hypoglycemia: repeat access to prehospital or emergency care. Prehosp Emerg Care. 2019;23:364-76.

5. Abarbanell NR. Is prehospital blood glucose measurement necessary in suspected cerebrovascular accident patients? Am J Emerg Med. 2005;23:823-27.

6. Known KT, Tsai VW. Metabolic emergencies. Emerg Med Clin N Am. 2007;25:1041-60.

7. Cryer PE, Davis SN, Shamoon HS. Hypoglycemia in diabetes. Diabetes Care. 2003;26:1902-12.

8. Tesfaye N, Seaquist ER. Neuroendocrine responses to hypoglycemia. Ann N Y Acad Sci. 2010;1212:12-28.

9. Tran C, Gariani K, Herrmann FR, Juan L, Philippe J, Rutschmann OT, et al. Hypothermia is a frequent sign of severe hypoglycaemia in patients with diabetes. Diabetes Metab. 2012;38:370-2.

10. Frienkel N, Metzger BE, Harris E, Robinson S, Mager M. The hypothermia of hypoglycemia. Studies with 2-deoxy-D-glucose in normal human subjects and mice. N Engl J Med. 1972;287:841-5.

11. Ikeda M, Matsunaga T, Irabu N, Yoshida S. Using vital signs to diagnosis impaired consciousness: cross observational study. BMJ. 2002;325:800-2.

12. Molnar GW, Read RC. Hypoglycemia and body temperature. JAMA. 1974;227:916-21. 
13. Wallgren UM, Bohm KEM, Kurland L. Presentations of adult septic patients in the prehospital setting as recorded by emergency medical services: a mixed methods analysis. Scand J Trauma Resusc Emerg Med. 2017;25:23.

14. Fujita Y, Tajimi K. Evaluation of intravenous access for shock and glucose administration for hypoglycemia patient by prehospital emergency life-saving technicians for first 6 months in AKITA. JJSEM. 2016;19:592-7.

15. Kikuchi Y, Nishiyama T, Shirotsuki T, Okada N, Andou Y. Assessment of the effectiveness of a protocol for prehospital glucose infusion at the Kobe City Fire Bureau. JJSEM 2018;21:504 - 12.

16. McCoy RG, Lipska KJ, Van Houten HK, Shah ND. Association of cumulative multimorbidity, glycemic control, and medication use with hypoglycemia-related emergency department visits and hospitalizations among adults with diabetes. JAMA Netw Open. 2020;3:e1919099.

17. Karter AJ, Warton EM, Lipska KJ, Ralston JD, Moffet HH, Jackson GG, Huang ES, Miller DR. Development and validation of a tool to identify patients with type 2 diabetes at high risk of hypoglycemia-related emergency department or hospital use. JAMA Intern Med. 2017;177:1461-70.

18. Takahashi T, Ito T, Takei T, Yagi K. A study of emergency visits for hypoglycemia cases. JJAAM. 2013;24:391-8.

19. Rincon F, Hunter K, Schorr C, Dellinger RP, Zanotti-Cavazzoni S. The epidemiology of spontaneous fever and hypothermia on admission of brain injury patients to intensive care units: a multicenter cohort study. J Neurosurg. 2014;121:950-60.

\section{Figures}




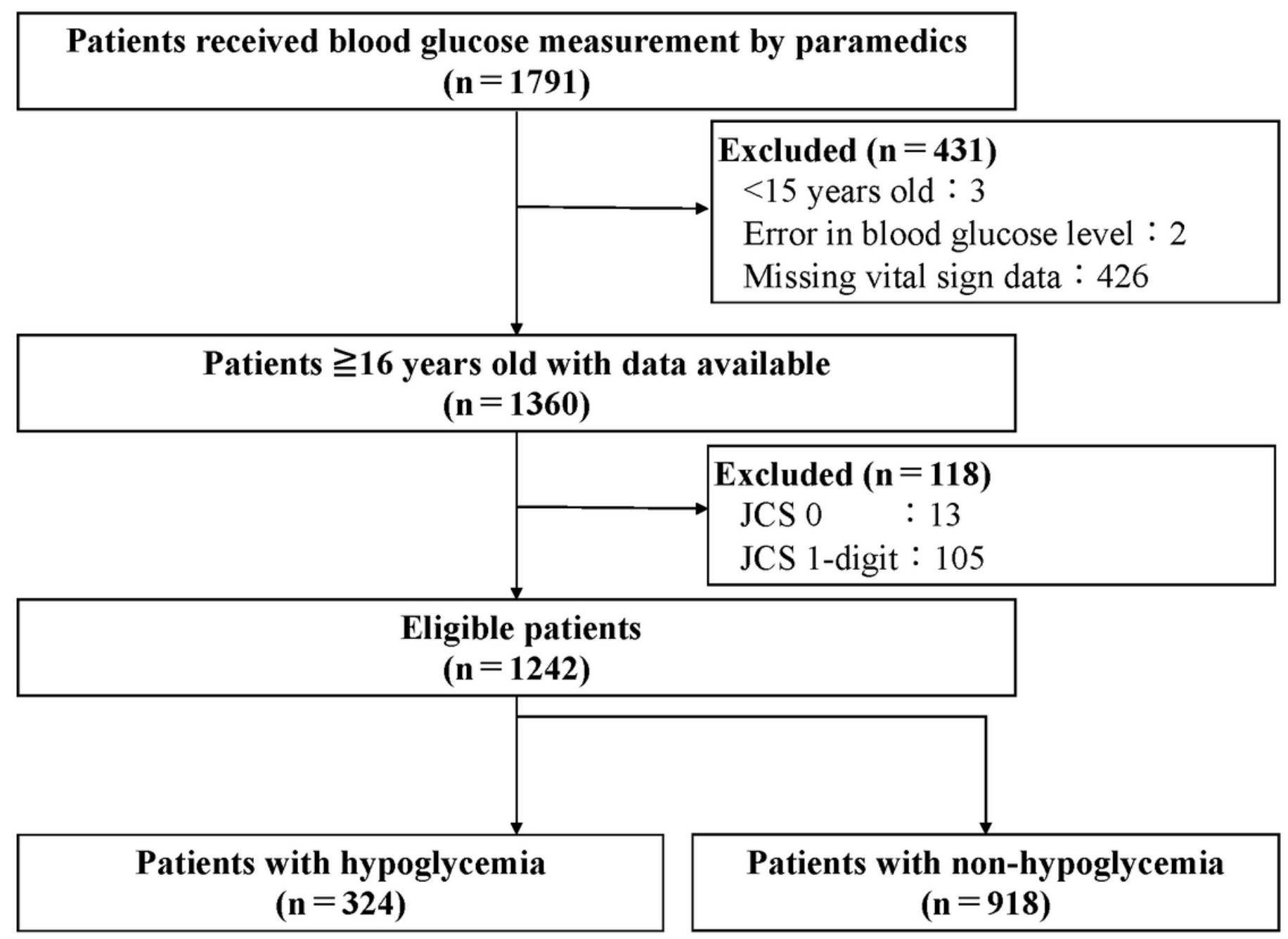

Figure 1

Study flow chart Of the 1791 patients, the data of 253 non-hypoglycaemic eligible patients is finally analysed to determine if vital signs could predict hypoglycaemia in pre-hospital settings in patients with altered mental status. 

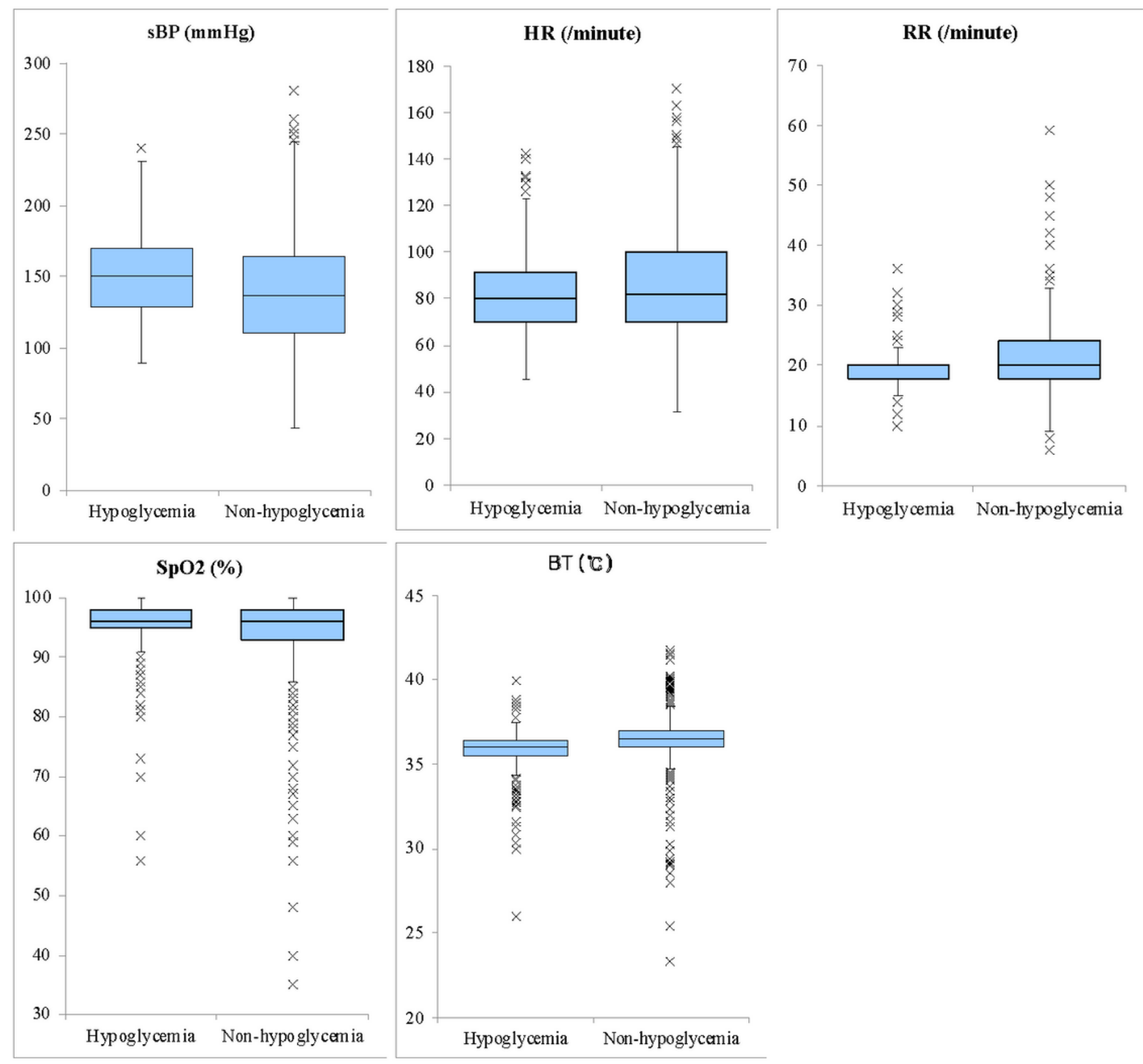

\section{Figure 2}

The distribution of each vital signs depending on the presence of hypoglycaemia When systolic blood pressure was less than $100 \mathrm{mmHg}$ and body temperature is more than $38^{\circ} \mathrm{C}$, the presence of hypoglycaemia is unlikely. sBP: systolic blood pressure; HR: heart rate; RR: respiratory rate; Sp02: oxygen saturation by pulse oximetry; BT: body temperature 


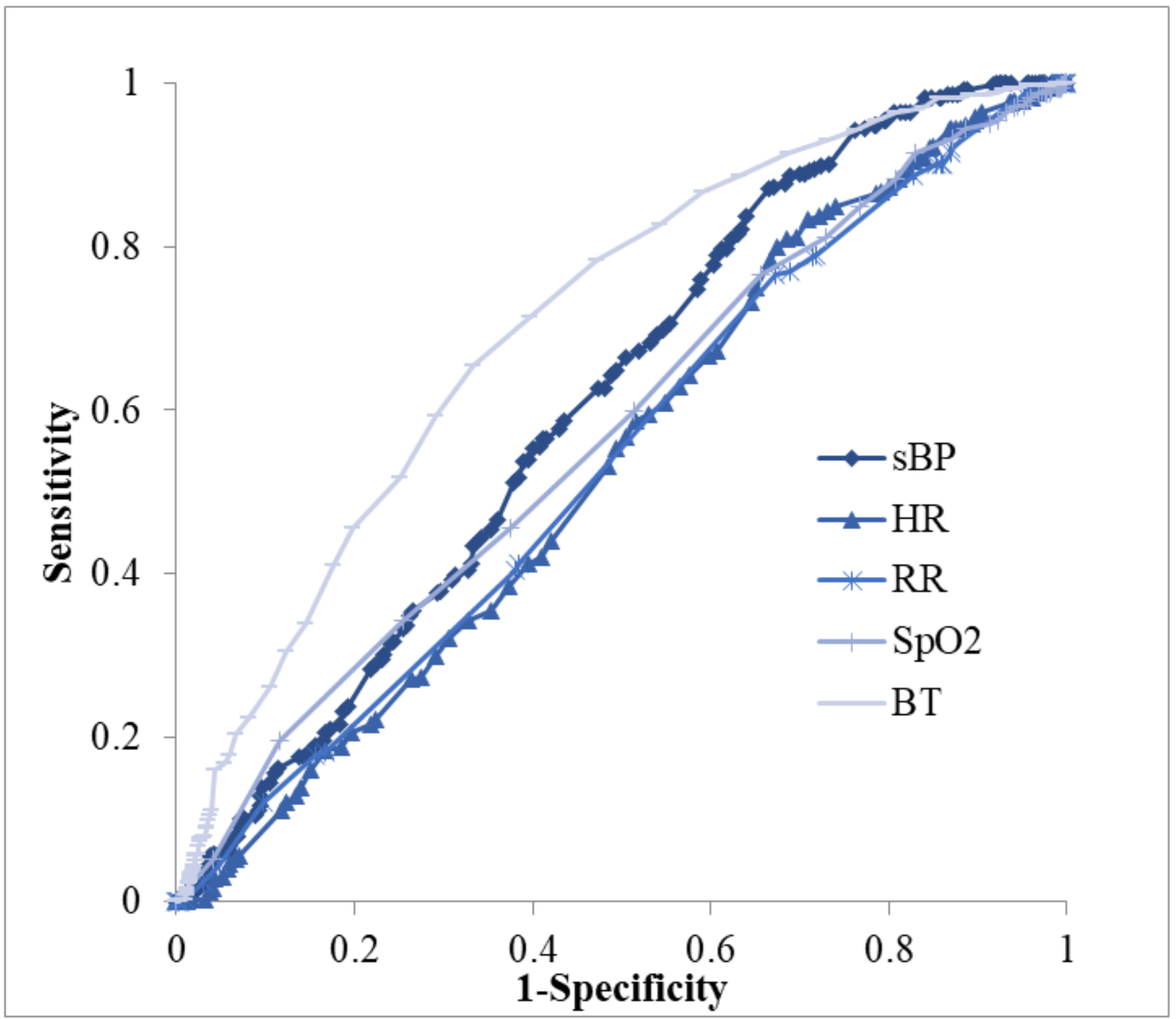

Figure 3

Receiver operating characteristic curve for each vital sign The sensitivity and specificity of each vital sign to predict hypoglycaemia was evaluated. sBP: systolic blood pressure; HR: heart rate; RR: respiratory rate; SpO2: oxygen saturation by pulse oximetry [SpO2]; BT: body temperature 